\title{
Analysis of MMSE-based anti-interference performance for MIMO-UWB systems
}

\author{
Biao Yan, Jiamin Wang, Dingping Guo, Saiyan Cai \\ School of Information Engineering \\ Yangzhou University \\ Yangzhou Jiangsu, China \\ byan@yzu.edu.cn
}

\begin{abstract}
The combinations of MIMO (Multiple-InputMultiple-Output) and UWB (Ultra-Wide-Band) systems greatly improve the performance of the UWB system. For the MIMO-UWB system, a new decoding scheme is proposed based on the STF (Space-Time-Frequency) coding and it can reduce the decoding complexity. The simulation results show that under the same SNR, the new method can obtain better performance of the bit error rate than the traditional ML decoding method.
\end{abstract}

\section{Keywords-MIMO-UWB; BER; MMSE}

\section{INTRODUCTION}

The ultra-wideband (UWB) system has many advantages, such as high transform rate, low-power, anti-multipath and anti-narrowband interference ability, and large capacity. It has been widely used in the wireless communications of high data rate and short range in recent years. In compliance with the FCC regulations, UWB is defined as nominally more than $500 \mathrm{MHz}$ bandwidth in the range from 3.1 to $10.6 \mathrm{GHz}$. Two standards proposed by IEEE 802.15.3a are MB-OFDM UWB and DS-UWB. In MB-OFDM scheme, the overall bandwidth of $7.5 \mathrm{GHz}$ is split into some smaller subbands of bandwidth 528MHz.

Multiple-input multiple-output (MIMO) technology can improve the data transfer rate, spectral efficiency, and transmission quality of the wireless communication systems, and also enhance the capacity of the system. Therefore, the combination of MIMO technology with UWB can effectively increase the data transfer rate and spectral efficiency.

There are a lot of literatures for MIMO-UWB systems [12] and the use of multi-antenna spatial diversity techniques greatly improve the information transfer rate. One of the key technologies of the MIMO-UWB systems is channel coding technique. In $[3,4]$, the anti-interference ability of the system is improved through space-time-frequency (STF) coding based on $\Delta-K$ and $S-V$ channel models, but it adds the system complexity of the transmitter and receiver sides under the influence of system dimension. The pre-decoding scheme based on the dual-carrier modulation greatly simplifies the system complexity [5], but it reduces the spectral efficiency of the system. The STF coding methods based on narrowband are researched in [6,7].

In this paper, the bit error rate (BER) performance of the MIMO-UWB system is analyzed based on the S-V channel model through a simple STF coding scheme, and a low complexity MMSE algorithm for decoding is also proposed. Theoretical analysis and simulation results show that in comparison with a traditional ML decoding method, the new decoding method further reduces the BER of the MIMO MB-OFDM UWB system.

\section{MIMO MB-OFDM UWB SYSTEM MODEL}

\section{A. MIMO MB-OFDM UWB system}

The system with $M_{t}$ transmit and $M_{r}$ receive antennas is considered and there are $N_{s}$ date streams that are modulated on $\mathrm{N}$ subcarriers. A MIMO MB-OFDM UWB system is shown in Figure1.

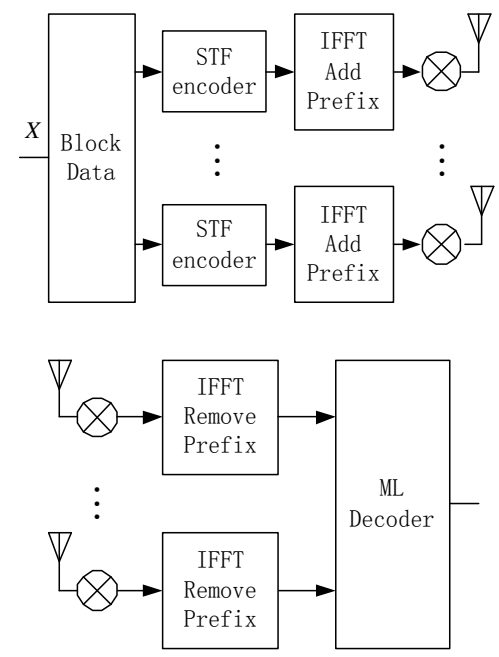

Figure 1. System model for MIMO MB-OFDM UWB

The OFDM symbol transmitted from the i-th transmit antenna is given by [3]

$$
s_{i}(t)=\sum_{k=0}^{K-1} \operatorname{Re}\left\{x_{i}^{u}\left(t-k T_{S Y M}\right) \exp \left(j 2 \pi f_{c}^{u} t\right)\right\}
$$

where $\mathrm{K}$ is the number of OFDM blocks for each antenna, $x_{i}^{u}(t)$ is the baseband OFDM signal to be transmitted by the 
i-th transmit antenna at the u-th OFDM block, it can be expressed as

$$
x_{t}^{u}(t)=\sqrt{\frac{E}{N}} \sum_{n=0}^{N-1} d_{i}^{u}(n) \exp \left\{(j 2 \pi n \Delta f)\left(t-T_{C P}\right)\right\}
$$

where $\mathrm{N}$ is the subcarrier number of an OFDM symbol.

\section{B. Channel Model}

Issued by the Sub-Committee of the IEEE channel model based on cluster approach model (SV) UWB system, the channel impulse response can be shown as

$$
h(t)=X \sum_{n=1}^{N} \sum_{k=1}^{K(n)} \alpha_{n k} \delta\left(t-T_{n}-\tau_{n k}\right)
$$

Therefore, the channel impulse response from i-th transmit antenna to $\mathrm{j}$-th receive antenna in the MIMO-UWB system can be described as [4]

$$
h_{i j}^{u}(t)=A \sum_{l-0}^{L-1} \sum_{k=0}^{K-1} \alpha_{k l}^{u} \delta\left(t-T_{l}-\tau_{k l}\right)
$$

where $\mathrm{A}$ is the amplitude gain of the channel, $\alpha_{k l}^{u}$ is k-th multipath coefficient in l-th cluster during u-th OFDM block. $T_{l}$ represents the arrival time of l-th cluster and $\tau_{k l}$ denotes the delay of k-th multipath contribution relative to the l-th cluster.

We can obtain the channel frequency response from the ith transmit antenna to the $\mathrm{j}$-th receive antenna at the n-th subcarrier in the u-th OFDM block, which is given by

$$
H_{i j}^{u}(n)=\sum_{l=0}^{L-1} \sum_{k=0}^{K-1} \alpha_{k l}^{u} \exp \left(-j 2 \pi n \Delta f\left(T_{l}+\tau_{k l}\right)\right)
$$

For the $S-V$ model and observations, the multipath components from a pulse usually reach a receiver in the form of a cluster. The arrival time of clusters and multipath components are simulated as the Poisson distribution with $\Lambda$ rate and $\lambda$ rate respectively. The average power delay profile is

$$
\Omega_{k, l}^{u}=E\left[\left|\alpha_{k, l}^{u}\right|^{2}\right]=\Omega_{0} \exp \left(-T_{l} / \Gamma\right) \exp \left(-\tau_{k, l} / \gamma\right),
$$

where $\Omega_{k, l}^{u}$ is the mean energy of the first cluster pulse in the first path, $\Gamma$ is the power decay factor of a cluster and $\gamma$ is the power decay factor within cluster. $\sum_{l=0}^{L-1} \sum_{k=0}^{K-1} \Omega_{k, l}^{u}=1$.

\section{MIMO MB-OFDM UWB system}

At transmit antennas, the data $\mathrm{X}$ are encoded via a STF coding, each STF codeword matrix D of size $N \times M_{t} M_{b}$ can be expressed as $D=\left[D^{1}, D^{2}, \ldots, D^{M_{b}}\right]$, where $D^{u}=\left[d_{1}^{u}, d_{2}^{u}, \ldots, d_{M_{t}}^{u}\right]$, and $d_{i}^{u}=\left[d_{i(0)}^{u}, d_{i(2)}^{u}, \ldots, d_{i(N-1)}^{u}\right]^{T}$. Then during the u-th OFDM block period, the complex symbol $d_{i}^{u}(n)$ is transmitted over subcarrier $\mathrm{n}$ by the i-th transmit antenna, after applying OFDM modulation, which are transmitted simultaneously from all over $M_{t}$ transmit antennas.

Provided that the system synchronization has been achieved in the receivers, the receive signal at the $\mathrm{j}$-th receive antenna in the matrix form is given by

$$
Y_{j}=\sqrt{E / M_{t}} D H_{j}+Z_{j}
$$

where $\mathrm{E}$ is the average energy per transmitted symbol, $\mathrm{D}$ is an $M_{b} N \times M_{b} N M_{t}$ data matrix constructed from the STF codeword D, $D=\left[D_{1}, D_{2}, \ldots, D_{M_{t}}\right] \quad$ and $D_{i}=\operatorname{diag}\left(d_{i}(0), d_{i}(1), \ldots, d_{i}\left(M_{b} N-1\right)\right)$. Both $Z_{j}$ and $Y_{j}$ are Gaussian white noise signal matrix with same size. Finally, through the ML criterion, the signals $Y_{j}$ in $M_{r}$ receive antennas can be obtained when $D=\hat{D}$ is satisfied. Here, $\hat{D}$ is

$$
\widehat{D}=\arg \min _{D} \sum_{j=1}^{M_{r}}\left\|Y_{j}-\sqrt{E / M_{t}} D H_{j}\right\|_{F}^{2}
$$

\section{STF CODING AND DECODING}

\section{A. STF Codes}

Given that $M_{b}=2, M_{t}=2, M_{r}=1$, and $N=2$, the STF code with two OFDM blocks can be expressed as [6]

$$
D_{S T F}=\left[\begin{array}{cc}
d_{1,1} & d_{2,1} \\
-d_{2,1}^{*} & d_{1,1}^{*} \\
d_{1,2} & d_{2,2} \\
-d_{2,2}^{*} & d_{1,2}^{*}
\end{array}\right]
$$

where $d_{i}=\left(d_{i, 1}, d_{i, 2}\right)^{T}=\left(d_{2 i-2}, d_{2 i-1}\right)^{T}$. The system channel matrix can be shown as

$$
\begin{gathered}
H(h)=\left[H_{1}(h) H_{2}(h)\right] \\
H_{1}=\left[\begin{array}{cc}
H_{1,1}(0) & 0 \\
H_{2,1}^{*}(1) & 0 \\
0 & H_{1,1}(2) \\
0 & H_{2,1}^{*}(3)
\end{array}\right]
\end{gathered}
$$




$$
H_{2}=\left[\begin{array}{cc}
H_{2,1}(0) & 0 \\
-H_{1,1}^{*}(1) & 0 \\
0 & H_{2,1}(2) \\
0 & -H_{1,1}^{*}(3)
\end{array}\right]
$$

\section{B. ML Algorithm}

According to ML estimation algorithm, we can get

$$
\widehat{d}_{M L}=\arg \min \|H \cdot d-y\|^{2}
$$

If $H^{H} H=\alpha I$, the maximum likelihood estimation of $d$ can be indicated as

$$
\begin{aligned}
& \widehat{d}_{M L}=\arg \min \left\|H^{H} y-\alpha \sqrt{E / M_{t}} \cdot d\right\|^{2} \\
& =\arg \min \left\{\alpha\left[\left\|H^{H} y-d\right\|^{2}+(\alpha-1)\|d\|^{2}\right]+(1-\alpha) y^{H} H H^{H} y\right\} \\
& =\arg \min \left\{\left\|H^{H} y-d\right\|^{2}+\left(\alpha \sqrt{E / M_{t}}-1\right)\|d\|^{2}\right\} \\
& =\arg \min \left\|H^{H} y-d\right\|^{2}
\end{aligned}
$$

\section{MMSE Algorithm}

For convenient discussion, provided that $y=H d+n$ meets, we can acquire by MMSE-BLE algorithm [8]

$$
\widehat{d}=\left(H^{H} R_{n} H+R_{d}^{-1}\right)^{-1} H^{H} R_{n}^{-1} y
$$

where $R_{d}=E\left\{d d^{H}\right\}$ is covariance matrix of the code vector d, and $R_{n}=E\left\{n n^{H}\right\}$ is covariance matrix of the noise vector. If $R_{d}=I$ and $\mathrm{n}$ is white noise, equation (14) can be simplified as

$$
\widehat{d}=\left(H^{H} H+\sigma^{2} I\right)^{-1} H^{H} y
$$

\section{SYSTEM SIMULATION AND ANALYSIS}

We can design the system simulation parameters by referring to the UWB channel model parameters that IEEE sets down (shown in Table 1), and implement the simulation of the system BER under CM1-CM4 channels respectively. In the systems with QPSK and BPSK modulation, the subcarrier number $\mathrm{N}=128$, the system bandwidth $528 \mathrm{MHz}$ and the guard interval $\mathrm{Ng}=16$, we suppose that the carriers and symbols have strict synchronization.

Figures 2 and 3 show the system BER curves in different channels respectively. We can see from them that as the antenna number adds, the system BER performance under the both channels enhances. The performance of the MIMOUWB system is superior to that of the SISO-UWB system obviously. For example, when the mean square error is equal to $10^{-4}$, the UWB system with two antennas increases signal-noise ratio ( SNR ) $5 \mathrm{~dB}$ relative to one with singleantenna.

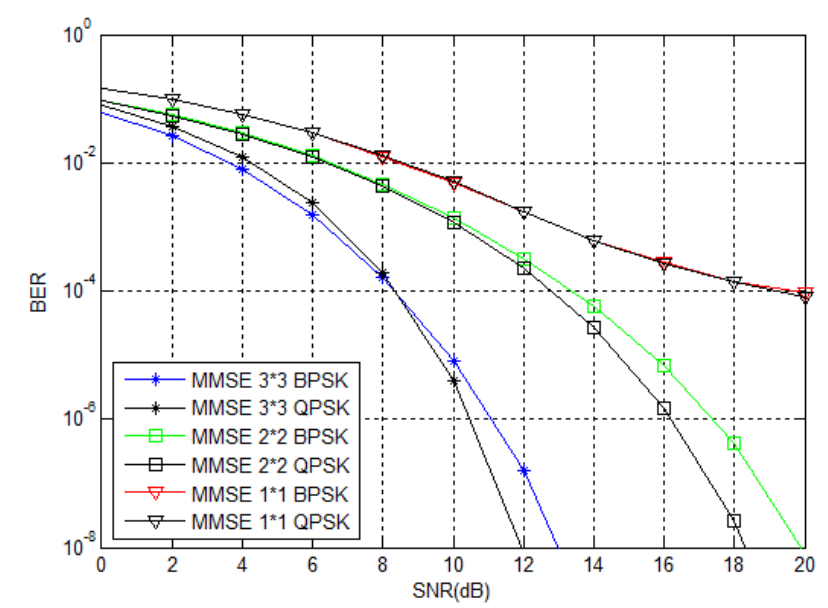

Figure 2. BER of the CM1channel

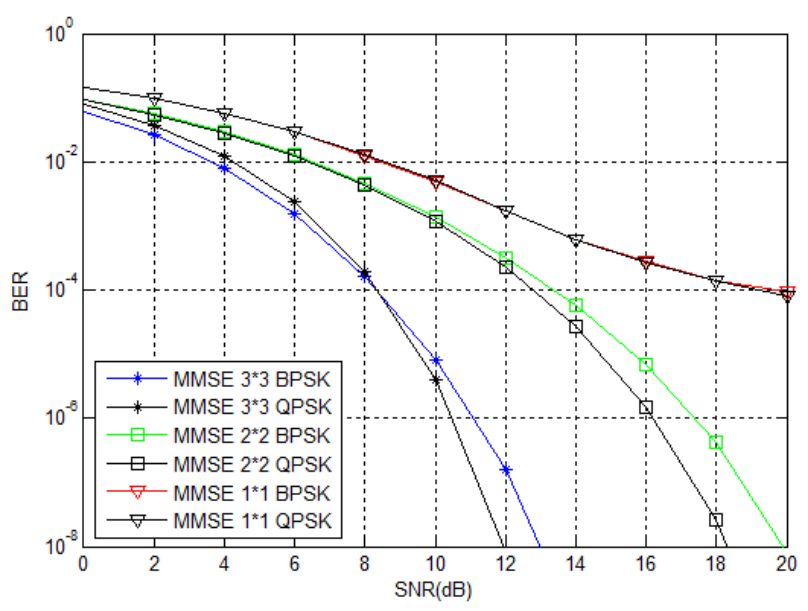

Figure 3. BER of the CM4 channel

Figure 4 shows the BER performance comparison of the MIMO-UWB systems when the different decoding methods are used. It can be observed that as SNR advances, the system BER under two decoding criterions drops gradually. The system performance with the MMSE algorithm becomes better for comparison with that with the ML algorithm at the same SNR. But the decoding complexity of the MMSE algorithm becomes lower for contrast with that of the ML algorithm. 


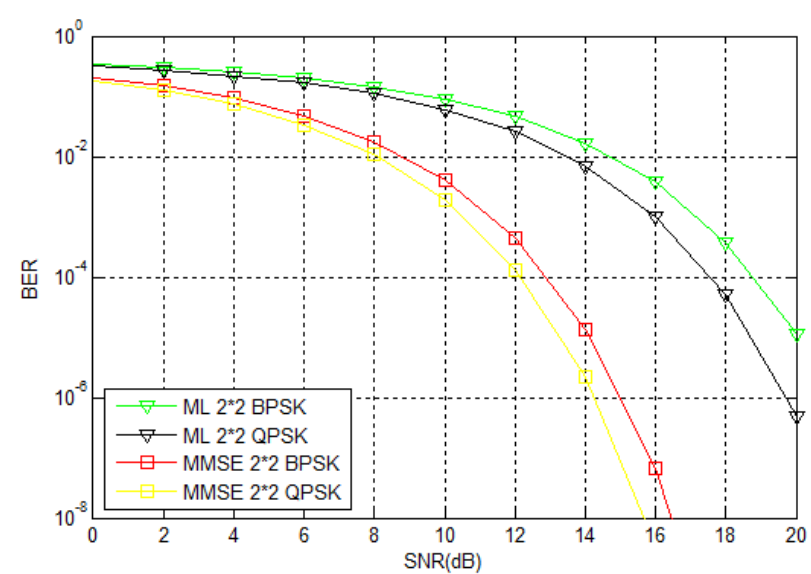

Figure 4. System BER of the MMSE and ML estimation

\section{CONCLUSION}

The anti-interference performance of the MIMO-UWB system is investigated in this paper. For the anti-interference performance of the UWB system, the performance comparison between the system with multiple antennas and one with single antenna is conducted. Through optimizing the STF coding and decoding ways, we introduce the MMSE algorithm which has lower complexity relative to ML algorithm. The simulation results indicate that the MMSE algorithm exceeds the ML algorithm in the anti-interference and moreover, has lower decoding complexity.

\section{REFERENCES}

[1] E. Dimitrov, T. Kasiser,“Advanced MIMO VHDR MB-OFDM approaches," IEEE International Conference on Ultra-Wideband, 2008,pp.51-54.

[2] E. K. A. Ampoma, T. R. Rao, and V. A. Labay, "Capacity \& performance issues in a MIMO based MB-OFDM ultrawide band communication system," $2^{\text {nd }}$ International Conference on Adaptive Science \& Technology (ICAST 2009), 2009,pp.432-439.

[3] W. P. Siriwongpairat, Weifeng Su, M. Olfat, and K. J. R. Liu, "Multiband-OFDM MIMO coding framework for UWB communication systems," IEEE Transactions on Signal Processing, 2006, 54(1):214-224.

[4] J. Chusing, L. Wuttisttikulkij, and S. Segkhoontod,“Achieving rate two Space-Time-Frequency codes for multiband UWB-MIMO communication systems using rotated multidimensional modulation,” Fifth Annual Conference on Communications Networks and Services Research (CNSR’07), 2007,pp.294-301.

[5] Tien Hoa Nguyen, Thanh Hieu Nguyen, and Van Tuyen Tran,etc,“A scheme of dual carrier modulation with soft-decoding for MB-OFDM MIMO systems,” 2011 International Conference on Advanced Technologies for Communications (ATC), 2011,pp.220-223.

[6] Wei Duan, Ying Guo, and Moon Ho Lee,"High-rate full-diversity space-time-frequency codes with PIC decoding,” 2011 International Conference on Electrical and Control Engineering (ICECE), 2011,pp.5956-5961.

[7] Philip R. Botha, Daniel J. Louw and B. T. Maharaj,“Achievable diversity limits in a quantized MIMO-OFDM linear pre-coded system," $20105^{\text {th }}$ IEEE International Symposium on Wireless Pervasive Computing (ISWPC), 2010,pp.455-459.

[8] Le Chung, E. Dimitrov, S. Moghaddamnia, and T. Kaiser,"Performance investigation of MMSE-based interference alignment for multiuser MIMO UWB,” 2011 IEEE International Conference on Ultra-Wideband (ICUWB), 2011,pp.170-174.

TABLE I. UWB CHANNEL MODEL PARAMETERS

\begin{tabular}{|c|c|c|c|c|c|c|c|}
\hline Scheme & $\Lambda$ (1/ns) & $\lambda(1 / \mathrm{ns})$ & $\Gamma$ & $\gamma$ & $\sigma_{\xi}(\mathrm{dB})$ & $\sigma_{\varsigma}(\mathrm{dB})$ & $\sigma_{g}(\mathrm{~dB})$ \\
\hline CM1 & 0.0233 & 2.5 & 7.1 & 4.3 & 3.3941 & 3.3941 & 3 \\
CM2 & 0.4 & 0.5 & 5.5 & 6.7 & 3.3941 & 3.3941 & 3 \\
CM3 & 0.0667 & 2.1 & 14 & 7.9 & 3.3941 & 3.3941 & 3 \\
CM4 & 0.0667 & 2.1 & 24 & 12 & 3.3941 & 3.3941 & 3 \\
\hline
\end{tabular}

